\title{
Combinatorial properties of permutation tableaux
}

\author{
Alexander Burstein 啡 and Niklas Eriksen非 \\ ${ }^{1}$ Department of Mathematics, Howard University, Washington, DC 20059, USA \\ ${ }^{2}$ Department of Mathematical Sciences, Göteborg University and Chalmers University of Technology, SE-412 96 \\ Göteborg, Sweden.
}

\begin{abstract}
We give another construction of a permutation tableau from its corresponding permutation and construct a permutation-preserving bijection between 1-hinge and 0-hinge tableaux. We also consider certain alignment and crossing statistics on permutation tableaux that have previously been shown to be equidistributed by mapping them to patterns in related permutations. We give two direct maps on tableaux that prove the equidistribution of those statistics by exchanging some statistics and preserving the rest. Finally, we enumerate some sets of permutations that are restricted both by pattern avoidance and by certain parameters of their associated permutation tableaux.
\end{abstract}

Résumé. Nous donnons une nouvelle construction d'un tableau de permutation à partir de la permutation correspondante. Nous construisons ensuite une permutation qui préserve la bijection entre un tableau charnière 1 et tableau charnière 0 . Nous considérons également certaines statistiques sur les alignements et croisements dans les tableaux de permutations. L'équidistribution de ces données statistiques est connue, et donnée par le biais d'une application très compliquée associant les alignements et croisements des tableaux a des motifs des permutations correspondantes. Nous constuisons deux involutions définies sur les tableaux qui démontrent l'équidistribution des statistiques en échangeant certaines données tout en préservant d'autres. Enfin, nous dénombrons quelques ensembles de permutations définis non seulement par l'absence de certains motifs mais aussi par certains paramètres issus des tableaux de permutations.

Keywords: permutation tableaux, permutation patterns

\section{Introduction}

Permutation tableaux are combinatorial objects that are in bijection with permutations. They originally turned up in the enumeration of totally positive Grassmannian cells [8, 10]. Permutation tableaux have then been studied either in their own right [1, 9] to produce enumeration results for permutations, or in connection with the PASEP model in statistical mechanics [3, 5, 6].

\footnotetext{
${ }^{\dagger}$ Research supported in part by NSA Young Investigator Grant H98230-06-1-0037.

‡Email: aburstein@howard.edu, alex@alexanderburstein.com.

§Email: ner@math.chalmers.se. 


\begin{tabular}{|l|l|l|l|}
\hline 0 & 1 & 0 & 1 \\
\hline 1 & 1 & 0 & 1 \\
\hline 1 & 1 & 1 & 2 \\
\hline 0 & 1 & 1 & 2 \\
\hline
\end{tabular}

\begin{tabular}{|l|l|l|l|}
\hline 0 & 1 & 0 & 1 \\
\hline 1 & 1 & 0 & 1 \\
\hline 1 & 1 & 1 & \multicolumn{1}{|}{} \\
\cline { 1 - 2 } 0 & 1 & 1 & \multicolumn{1}{|c}{} \\
\cline { 1 - 2 } & &
\end{tabular}
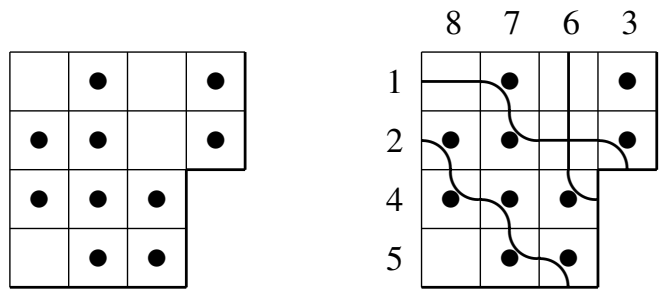

Fig. 1: Permutation tableaux (1-hinge) of $\pi=36187425$. To the right is illustrated $\pi(1)=3, \pi(2)=6$ and $\pi(6)=4$

A permutation tableaux $\mathcal{T}$ is usually defined as a $k \times(n-k)$ array filled with zeroes, ones and twos such that the cells filled with zeroes and ones form a Young tableau $Y_{\lambda}$ of an integer partition $\lambda$ such that $n-k=\lambda_{1} \geq \lambda_{2} \geq \ldots \geq \lambda_{k} \geq 0$ (note that zero parts are allowed), and such that these rules are obeyed:

(column): Each column in the tableau contains at least one 1.

(1-hinge): Each cell in the Young tableau $Y_{\lambda}$ with a 1 above in the same column and to its left in the same row must contain a 1 .

Equivalently, one forgets about the twos and considers only the Young tableau of zeroes and ones, which are sometimes encoded as blanks and bullets, respectively (see Figure 1 1 . The shape of $\mathcal{T}$ is the integer partition $\operatorname{sh}(\mathcal{T})=\lambda$ and its length is the number of parts in $\lambda, \ell(\mathcal{T})=k$.

The second rule above can, however, take several forms. An alternative [1] is this:

(0-hinge): Each cell in the Young tableau $Y_{\lambda}$ with a 1 above in the same column and to its left in the same row must contain a 0 .

In this paper, we generalize these hinge rules:

(0/1-hinge): Given any partition $\mu \leq \lambda$ (i.e. $\mu_{i} \leq \lambda_{i}$ for all $i \geq 0$ ), each cell in the Young tableau $Y_{\lambda}$ with a 1 above in the same column and to its left in the same row must contain a 1 if the cell is in $\mu$ and a 0 otherwise.

The 0/1-hinge rule specializes to the 0 -hinge rule for $\mu=\emptyset$ an the 1-hinge rule for $\mu=\lambda$. We let $\mathfrak{T}_{n}$ denote the set of 1-hinge permutation tableaux $\mathcal{T}$ such that, with $\operatorname{sh}(\mathcal{T})=\lambda, \lambda_{1}+\ell(\mathcal{T})=n$.

Permutation tableaux have their name from a natural bijection $\Phi: \mathfrak{T}_{n} \rightarrow \mathfrak{S}_{n}$ between (1-hinge) permutations tableaux and permutations [9]. Let $n=\lambda_{1}+k$. Label the south-east boundary of the tableau with 1 to $n$, starting in the north-east corner, and extend these labels to the rows and columns they belong to. Then, starting at the top of a column (or the left of a row) labelled $i$, we follow the zig-zag path obtained by bouncing right or down every time we hit a 1 . If the label of the exit is $j$, we put $\pi(i)=j$. This is illustrated to the far right in Figure 1 .

There are several important statistics of $\pi=\Phi(\mathcal{T})$, typically related to the order relation between a position $i$ and its letter $\pi(i)$ which are easily deduced from $\mathcal{T}$. The weak excedances (positions $i$ such that $\pi(i) \geq i$ ) are given by the row labels and the deficiences (the opposites of weak excedances, that is 
positions $i$ such that $\pi(i)<i$ ) are given by the column labels. Fixed points correspond to empty rows. We let wex $(\pi)$ denote the number of weak excedances in $\pi$ and conclude that wex $(\pi)=k$. We also note that a bijection between permutations tableaux and permutations with $n-k$ descents (elements $\pi(i)$ such that $\pi(i)>\pi(i+1))$ has been proposed [4], which amongst other things re-proves that descents and deficiencies are equidistributed.

The inverse $\Gamma=\Phi^{-1}: \mathfrak{S}_{n} \rightarrow \mathfrak{T}_{n}$ of the presented bijection is less natural, and several algorithms describing $\Gamma$ have been proposed [1, 9]. We continue this tradition with another algorithm which we consider simpler than the previous ones. It is presented in Section 2 together with the presentation of 0/1-hinge tableaux.

Further, we study two bijections on tableaux. Alignment and crossing statistics on permutations have been proposed by Corteel [3], and they have natural interpretations on tableaux. First we give in Section 3 a direct description on tableaux for the bijection between tableaux that belong to permutation $\pi$ and $\operatorname{irc}(\pi)$, where irc is the inverse of the reversal of the complement. This exchanges $A_{\mathrm{EN}}$ and $A_{\mathrm{NE}}$ statistics and preserves statistics wex, $A_{\mathrm{EE}}, A_{\mathrm{NN}}, C_{\mathrm{EE}}, C_{\mathrm{NN}}$. Then we give a simple bijection on tableaux that preserves the $A_{\mathrm{EN}}$ and $A_{\mathrm{NE}}$ statistics, and exchange the $A_{\mathrm{NN}}+A_{\mathrm{EE}}$ and $C_{\mathrm{NN}}+C_{\mathrm{EE}}$ statistics. Definitions and algorithm are presented in Section 4

Pattern avoidance (or pattern statistics in general) and tableau restrictions do not always combine easily and naturally. Patterns deal with comparisons of different letters in a permutation, in particular, they are better suited for considering descents and inversions. Permutation tableaux, on the other hand, naturally emphasize excedances, fixed points and deficiencies, i.e. comparisons of letters with their positions. Thus, permutation tableaux are useful in considering pattern-restricted sets when the information about descents and inversions can be translated into information about weak excedances and deficiencies. A good example of such a situation is [2] where alternating permutations (descent-related objects) with the maximum number of fixed points (excedance-related property) are considered.

In a 1-hinge tableau $\mathcal{T}$, those ones that are not forced by the 1-hinge rule are called essential. We conclude this article in Section 5 by giving some enumeration results on sets of permutations avoiding pairs of patterns of length 3 and whose associated tableaux (via maps $\Phi$ and $\Gamma$ ) have the maximum number of essential ones. The eventual goal of this undertaking is to refine pattern-occurrence statistics with respect to the number of essential ones in the associated tableaux.

\section{Combining 1-hinge and 0-hinge}

An important property of 1-hinge permutation tableaux is that every zero has a clear view (only zeroes) to its left or above it. We will use this property in two ways. First, we give a new algorithm for computing the 1-hinge tableaux of a permutation, and then we show that the 1-hinge and 0-hinge tableaux of a permutation are connected via a series of mixed hinge tableaux.

In a tableau $\mathcal{T}$, two paths are said to meet at a cell if each of these paths enters the cell. If the cell contains a zero, the paths will cross, and if it contains a one they will bounce. By the 1-hinge rule, two paths can only cross at their first meet.

Definition 2.1 In a tableau $\mathcal{T}$ of $\pi$, we have columns $c_{i}(\mathcal{T}), 1 \leq i \leq \lambda_{1}$ and rows $r_{i}(\mathcal{T}), 1 \leq i \leq n-\lambda_{1}$. When no confusion can arise, we write $c_{i}=c_{i}(\mathcal{T})$ and $r_{i}=r_{i}(\mathcal{T})$. Further, let ent $\left(c_{i}\right)$ be the label of column $c_{i}$ and let $\operatorname{ext}\left(c_{i}\right)=\pi\left(\operatorname{ent}\left(c_{i}\right)\right)$. We call these entry and exit labels of column $c_{i}$. Similar definitions apply for the rows. The row (resp. column) number with exit label $j$ is denoted $\operatorname{row}(j)$ (resp. $\operatorname{col}(j)$ ). In other words, $\operatorname{col}(j)=i \Leftrightarrow \operatorname{ext}\left(c_{i}\right)=j$. 
The initial zeroes in a column (or row) are the zeroes in that column (row) that have no ones above (to the left). The number of initial zeroes in a column or row is denoted $z\left(c_{i}\right)$ and $z\left(r_{i}\right)$, respectively, and similarly $t\left(c_{i}\right)$ and $t\left(r_{i}\right)$ for the number of twos in the column or row.

The exit labels of rows are the weak excedance letters and the exit labels of columns are the deficiency letters.

We note that information on the number of initial zeroes in all rows and columns completely determines $\mathcal{T} \in \mathfrak{T}_{n}$, since each zero is an initial zero of some row or column. The initial zeroes also determines the essential ones, and thus any 0/1-hinge tableau. Thus, to compute $\Gamma(\pi)$ we need only determine the initial zeroes. To accomplish this, only the relative order of the exit labels of rows and columns are important.

Definition 2.2 For a row $r_{i}$ in $\mathcal{T}$, we say that row $r_{i}$ has $m$ inversions if there are $m$ rows above with higher exit labels. Likewise, we say that column $c_{i}$ has $m$ inversions if there are $m$ columns to its left with lower exit labels. The number of inversions are denoted $\operatorname{inv}\left(r_{j}, \mathcal{T}\right)$ and $\operatorname{inv}\left(c_{j}, \mathcal{T}\right)$, respectively, or $\operatorname{inv}\left(r_{j}\right)$ and $\operatorname{inv}\left(c_{j}\right)$ for short.

As for usual permutation inversions, we need only to know the inversion numbers of all row (column) exit labels to compute their order.

Lemma 2.3 Consider a permutation tableau $\mathcal{T}$ with $\operatorname{sh}(\mathcal{T})=\lambda$. Let $\boldsymbol{r}$ contain the indices of the rows $r_{i}$ such that $\operatorname{ext}\left(r_{i}\right)>\operatorname{ext}\left(c_{j}\right)$ in increasing order. Then, $z\left(c_{j}\right)=\boldsymbol{r}_{\mathrm{inv}\left(c_{j}\right)+1}-1$. Similarly, letting c contain the columns $c_{i}$ such that $\operatorname{ext}\left(c_{i}\right)<\operatorname{ext}\left(r_{j}\right)$ in increasing order, with $\lambda_{j}+1$ appended at the end, we have $z\left(r_{j}\right)=\boldsymbol{c}_{\mathrm{inv}\left(r_{j}\right)+1}-1$.

Example 2.4 The lemma is best appreciated after an example. Consider the permutation $\pi=463785912$. Its tableau has shape $\lambda=\left(3^{5}, 2\right)$. The column exit labels are 215 (deficiency letters read from right to left) and the row exit labels are 463789 (weak excedance letters read from left to right).

Now, for the third column we get $\boldsymbol{r}=(2,4,5,6)$ and hence $z\left(c_{3}\right)=\boldsymbol{r}_{\text {inv }\left(c_{3}\right)+1}-1=\boldsymbol{r}_{2+1}-1=$ $5-1=4$. For $c_{2}$ and $c_{1}$, we find no inversions, and the first entry in $\boldsymbol{r}$ is 1 , yielding no initial zeroes.

For the rows, the only row with positive inversion number is $r_{3}$, with inv $v_{3}=2$. Computing $c=$ $\left(1,2, \lambda_{3}+1\right)$, we get $z\left(r_{3}\right)=c_{2+1}-1=\lambda_{3}+1-1=3$.

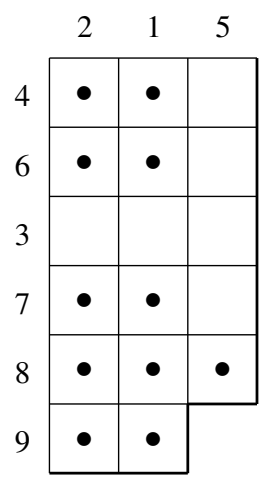

We are now ready to prove Lemma 2.3 
Proof: Assume that $z\left(c_{j}\right)=m$ and consider the array from $\mathcal{T}$ of the intersection of rows $r_{1}$ to $r_{m}$ and columns $c_{1}$ to $c_{j-1}$. The paths that enter the array must also exit the array. Those that exit horizontally will cross the path from $c_{j}$ and those that exit vertically will not, since paths starting at columns must cross at an initial zero of $c_{j}$, and paths starting at rows $r_{i}$ must cross at an initial zero of either $c_{j}$ or $r_{i}$, neither of which is possible with a vertical exit.

Thus, with $j-1$ vertical exits and $j-1-\operatorname{inv}\left(c_{j}\right)$ taken by columns, there must be $j-1-(j-$ $\left.1-\operatorname{inv}\left(c_{j}\right)\right)=\operatorname{inv}\left(c_{j}\right)$ rows with higher exit labels in the array. Since $r_{m+1}$ does not cross $c_{j}$ we find that $\boldsymbol{r}_{\mathrm{inv}\left(c_{j}\right)+1}=m+1$, which proves the lemma for columns. For rows, the situation is completely analogous, although it should be noted that rows without ones get the same number of initial zeroes as the length of the row.

We now turn to 0/1-hinge tableaux. We will prove that for any $\lambda<\mu$, the permutation $\pi \in S_{\mu}$ has a unique tableau such that the 1-hinge property is fulfilled on $\lambda$ and the 0-hinge property is fulfilled on $\mu / \lambda$. To prove this, we need a few definitions and a lemma. We also take the liberty of extending the function $\Phi: \mathfrak{T}_{n} \rightarrow \mathfrak{S}_{n}$ to allow any permutation tableau, in particular, a 0/1-hinge tableaux.

Definition 2.5 Let $S_{\lambda}$ denote the set of permutations $\pi$ such that $\operatorname{sh}(\Gamma(\pi))=\lambda$. Also, let $T_{\lambda}^{\mu}$ denote the set of tableaux $\mathcal{T}$ such that $\mathcal{T}$ fulfills the 1-hinge property on $Y_{\mu}$ and the 0-hinge property on $Y_{\lambda / \mu}$.

Lemma 2.6 Let $\pi \in S_{\lambda}$, and consider the integer partitions $\mu<\nu \leq \lambda$ such that $Y_{\nu / \mu}$ has exactly one cell. Further let $\mathcal{T} \in T_{\lambda}^{\nu}$ be a tableau such that $\Phi(\mathcal{T})=\pi$. Then, switching ones to zeroes and zeroes to ones on the first and last meetings in $\nu$ of the two paths that meet at $\nu / \mu$, we obtain a tableau $\mathcal{T}^{\prime} \in T_{\lambda}^{\mu}$ such that $\Phi\left(\mathcal{T}^{\prime}\right)=\pi$.

Proof: Let $i \rightarrow \pi(i)$ and $j \rightarrow \pi(j)$ be the paths that meet at $\nu / \mu$. It is obvious that $\Phi\left(\mathcal{T}^{\prime}\right)=\pi$ since each of the changes correspond to the transposition $(i j)$. Thus, what remains is to show that $\mathcal{T}^{\prime} \in T_{\lambda}^{\mu}$.

The paths can only cross at their first meet. Thus, if $\mathcal{T}(\nu / \mu)=0$, the first and last meetings coincide and no changes are made. The 0 -hinge property is trivially fulfilled on $\nu / \mu$, since only 1 s can violate it.

If $\mathcal{T}(\nu / \mu)=1$, the 0 -hinge property on $\lambda / \nu$ is still trivially fulfilled. On $\nu / \mu$, it is trivial if the value changes to 0 , and otherwise the 1 must be essential and cannot violate the 0 -hinge property. What remains is to show that changing the first meeting of paths does not violate the 1-hinge property. But changing the value at the first meeting to 0 is not a problem, since then the 1 was essential, and changing the value to 1 is legal too, by the following argument.

The new 1 must be essential, so assume without loss of generality that it is essential in its row. Then, any 0 s immediately to its right must have 0 s below in the row of the second meeting. These 0 s have a 1 to their left and hence can have no 1 above, so the 0 s immediately to the right of the new 1 do not violate the 1-hinge property.

Theorem 2.7 Let $\pi \in S_{\lambda}$ and let $\mu \leq \lambda$. Then, there is a unique tableau $\mathcal{T} \in T_{\lambda}^{\mu}$ such that $\Phi(\mathcal{T})=\pi$.

Proof: Given a tableau $\mathcal{T} \in T_{\lambda}^{\lambda}$, which is known to be unique from [9], we can use the algorithm of Lemma 2.6 to reduce the 1 -hinge part to $\mu$ cell by cell. Thus, it is clear that there is at least one $\mathcal{T} \in T_{\lambda}^{\mu}$ such that $\Phi(\mathcal{T})=\pi$.

At any given moment during the reduction there are usually several cells that can be moved from the 1-hinge area to the 0-hinge area. We need to show that no matter how we choose the order of them, we still end up with the same tableau. 
It is fairly easy to realize that for any two cells that can be chosen at the same time, the order of these is insignificant. The cells of their last meetings are on different rows and columns, so all paths through these cells are distinct. Thus, any changes induced by one of the cells will not affect the paths through another one of these cells, and hence will not affect the changes induced by that other cell.

By the strong convergence theorem [7], it suffices to show that any two moves that are valid at the same time commute and that the sequence of moves is finite for a game to have a unique end result. We have shown that any two moves commute and since the number of moves is $|\lambda / \mu|$, the uniqueness is proved.

We let $\Gamma_{\lambda}^{\mu}: S_{\lambda} \rightarrow T_{\lambda}^{\mu}$ map any permutation whose weak excedance pattern matches $\lambda$ to the tableau with the 1-hinge property on $\mu \leq \lambda$ and the 0 -hinge property on $\lambda / \mu$. Of course, its inverse is $\Phi$ restricted to $T_{\lambda}^{\mu}$.

\section{The irc map}

In [3], Sylvie Corteel defined the permutation statistics

$$
\begin{aligned}
& A_{\mathrm{EE}}(\pi)=|\{(i, j) \mid j<i \leq \pi(i)<\pi(j)\}|, \\
& A_{\mathrm{NN}}(\pi)=|\{(i, j) \mid \pi(j)<\pi(i)<i<j\}|, \\
& A_{\mathrm{EN}}(\pi)=|\{(i, j) \mid j \leq \pi(j)<\pi(i)<i\}|, \\
& A_{\mathrm{NE}}(\pi)=|\{(i, j) \mid \pi(i)<i<j \leq \pi(j)\}|, \\
& C_{\mathrm{EE}}(\pi)=|\{(i, j) \mid j<i \leq \pi(j)<\pi(i)\}|, \\
& C_{\mathrm{NN}}(\pi)=|\{(i, j) \mid \pi(i)<\pi(j)<i<j\}| .
\end{aligned}
$$

They are related to the 1-hinge permutation tableaux $\mathcal{T}=\Gamma(\pi)$ in the following way. Label the 0-cells in $\mathcal{T}$ with EN if the paths that cross there originated from one column and one row, NN if both these paths originated from columns and EE if both paths originated from rows. The 2-cells are labeled NE. Let $\operatorname{EN}(\mathcal{T})$ be the number of cells labelled EN in this labeling, and use similar notation for the other three labels. From [9] and [1] we know that

$$
\begin{aligned}
A_{\mathrm{EE}}(\pi) & =\mathrm{EE}(\mathcal{T}), \\
A_{\mathrm{NN}}(\pi) & =\mathrm{NN}(\mathcal{T}), \\
A_{\mathrm{EN}}(\pi) & =\mathrm{EN}(\mathcal{T}), \\
A_{\mathrm{NE}}(\pi) & =\mathrm{NE}(\mathcal{T}), \\
C_{\mathrm{EE}}(\pi)+C_{\mathrm{NN}}(\pi) & =\# \text { nontop } 1 \mathrm{~s}(\mathcal{T}) .
\end{aligned}
$$

The map irc $=i \circ r \circ c$ (inverse of reversal of complement) is known to preserve all alignment and crossing statistics on permutation except for exchanging statistics $A_{\mathrm{NE}}$ and $A_{\mathrm{EN}}$. We now show that the tableau of $\operatorname{irc}(\pi)$ can be easily computed from the tableau of $\pi$. The irc map on tableaux is also named $\operatorname{irc}(\mathcal{T})=\Gamma(\operatorname{irc}(\Psi(\mathcal{T})))$.

To simplify matters, we start by showing this bijection for quite restricted tableaux and successively remove the restriction until the general case is reached. The condition $A_{\mathrm{NE}}(\pi)=0$ simply means that the shape of $\Gamma(\pi)$ is a rectangle, and the condition $A_{\mathrm{EN}}(\pi)=0$ implies that the main diagonal of $\Gamma(\pi)$ (starting at the northwest corner) contains only ones. It is easy to see that if the main diagonal contains 
only ones then no path can cross it and hence $A_{\mathrm{EN}}(\pi)=\mathrm{EN}(\Gamma(\pi))=0$, and conversely, if a row (or column) has initial zeroes reaching the main diagonal, there are not enough rows (columns) to account for all these crossings, and hence $A_{\mathrm{EN}}(\pi)>0$.

Proposition 3.1 Let $\pi \in \mathfrak{S}_{n}$ and let $\mathcal{T}=\Gamma(\pi) \in \mathfrak{T}_{n}$ and assume $A_{\mathrm{EN}}(\pi)=A_{\mathrm{NE}}(\pi)=0$. To compute $\mathcal{T}^{\prime}=\operatorname{irc}(\mathcal{T})$, associate the number of initial zeroes to each row and column, and then order the columns by increasing $\operatorname{ext}\left(c_{i}\right)$ and the rows by decreasing $\operatorname{ext}\left(r_{i}\right)$.

Proof: If $\pi(i)=j$, then $\operatorname{irc}(\pi)(n+1-j)=n+1-i$. Thus, if $i$ is a weak excedance in $\pi$, then $n+1-j$ will be a weak excedance in $\operatorname{irc}(\pi)$. Assuming $A_{\mathrm{EN}}(\pi)=A_{\mathrm{NE}}(\pi)=0$, all these weak excedances map one of the $k=\operatorname{wex}(\pi)$ lowest elements on one of the $k$ highest elements. Since $i \leq k$ implies $n+1-i \geq n+1-k$ and vice versa, this holds for $\operatorname{irc}(\pi)$ as well, and hence $\operatorname{sh}\left(\mathcal{T}^{\prime}\right)=\operatorname{sh}(\mathcal{T})$. Further, for rectangular tableaux, we have $i=n+1-\operatorname{ent}\left(c_{i}\right)$, and for tableaux with only ones on the main diagonal, we have $z\left(c_{i}\right)=\operatorname{inv}\left(c_{i}\right)$.

Consider column $c_{i}(\mathcal{T})$. For each inversion $\pi(k)<\pi(i)<i<k$ we get an inversion in $\mathcal{T}^{\prime}$, since then $\operatorname{irc}(\pi)(n+1-\pi(k))<\operatorname{irc}(\pi)(n+1-\pi(i))<n+1-\pi(i)<n+1-\pi(k)$. All these inversions contribute to initial zeroes in the same column $c$ in $\mathcal{T}^{\prime}$. Since $\operatorname{ent}(c)=n+1-\pi(i)=n+1-\operatorname{ext}\left(c_{i}(\mathcal{T})\right)$, we get $c=c_{n+1-\operatorname{ent}(c)}=c_{\operatorname{ext}\left(c_{i}(\mathcal{T})\right)}$, which is the statement of the proposition. Rows are handled in a similar fashion.

Example 3.2 Consider the permutation $\pi=76485132$. Below we have its 1-hinge tableau $\mathcal{T}$ with rows and columns labeled with their exit labels $\operatorname{ext}\left(r_{i}\right)$ and $\operatorname{ext}\left(c_{i}\right)$, as well as the tableau $\mathcal{T}^{\prime}$ obtained by reordering columns and rows according to the proposition above. It is easy to check that $\Phi\left(\mathcal{T}^{\prime}\right)=\operatorname{irc}(\pi)=58746213$. Note that the number of initial Os for each exit label remains the same after reordering rows and columns.
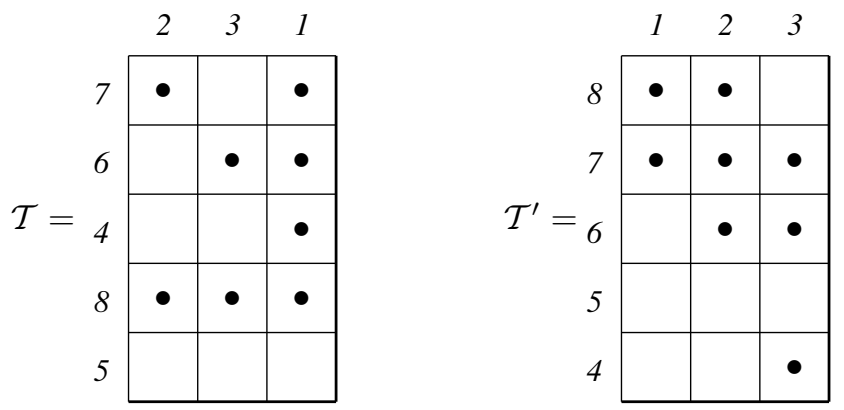

We now continue with permutations $\pi$ such that $A_{\mathrm{EN}}(\pi)>0$. The idea is to remove all EN cells, use the transformation for permutations with $A_{\mathrm{EN}}(\pi)=A_{\mathrm{NE}}(\pi)=0$ and then put them back as NE cells, which contain 2 s.

Proposition 3.3 Let $\pi \in \mathfrak{S}_{n}$ and let $\mathcal{T}=\Gamma(\pi) \in \mathfrak{T}_{n}$. Assuming $A_{\mathrm{NE}}(\pi)=0$, then the following algorithm will give $\mathcal{T}^{\prime \prime}=\operatorname{irc}(\mathcal{T})$. Let $\mathcal{T}^{\prime}$ have the same shape as $\mathcal{T}$ and let $z\left(c_{i}\left(\mathcal{T}^{\prime}\right)\right)=\operatorname{inv}\left(c_{i}(\mathcal{T})\right)$ for all columns (and similarly for the rows). Letting the column exit labels of $\mathcal{T}$ be $i_{1}<i_{2}<\ldots<i_{n-\operatorname{wex}(\pi)}$, compute $\mathcal{T}^{\prime \prime}=\operatorname{irc}\left(\mathcal{T}^{\prime}\right)$ and let $t\left(c_{j}\left(\mathcal{T}^{\prime \prime}\right)\right)=i_{j}-j$ for all $j$. 
Proof: The tableau $\mathcal{T}^{\prime}$ will have column and row exit labels in the same relative order as $\mathcal{T}$, since only EN cells are changed, but the column exit labels will be 1 to $n-\operatorname{wex}(\pi)$ and the row exit labels will be $n-\operatorname{wex}(\pi)+1$ to $n$. Let $k=\operatorname{wex}(\pi)$ and let the column exit labels in $\mathcal{T}$ be $i_{1}<i_{2}<\ldots<i_{k}$. Then, each column exit label is reduced by $a(j)=\operatorname{ext}\left(c_{j}(\mathcal{T})\right)-\operatorname{ext}\left(c_{j}\left(\mathcal{T}^{\prime}\right)\right)=i_{j}-j$. We define permutations $\sigma_{j}=\left(\left(i_{j}-a(j)\right)\left(i_{j}-a(j)+1\right) \ldots i_{j}\right)$ and obtain $\Psi\left(\mathcal{T}^{\prime}\right)=\sigma_{k} \ldots \sigma_{1} \pi$, since each $\sigma_{j}$ replaces the exit label $i_{j}$ with $j$, while maintaining the relative order of all other exit labels.

Further, $\operatorname{irc}\left(\mathcal{T}^{\prime}\right)$ is the tableau of

$$
\operatorname{irc}\left(\sigma_{k} \ldots \sigma_{1} \pi\right)=\operatorname{irc}(\pi) \operatorname{irc}\left(\sigma_{1}\right) \ldots \operatorname{irc}\left(\sigma_{k}\right),
$$

where $\operatorname{irc}\left(\sigma_{j}\right)=\left(\left(n+1-i_{j}\right)\left(n+1-i_{j}+1\right) \ldots\left(n+1-i_{j}+a(j)\right)\right)$. Multiplying from the right with $\left(\left(n+1-i_{k}\right)\left(n+1-i_{k}+1\right) \ldots\left(n+1-i_{k}+a(k)\right)\right)$ in a permutation circularly changes the positions of the letters in positions $n+1-i_{k}, n+1-i_{k}+1, \ldots, n+1-i_{k}+a(k)$, which is equivalent with introducing $a(k)$ twos in the rightmost column. Similarly, removing the other cycles introduce $a(j)$ in the $(k-j+1)$ th column from the right.

The irc bijection when $A_{\mathrm{NE}}(\pi)=0$ is clearly bijective. Thus, its inverse gives $\operatorname{irc}(\Gamma(\pi))$ when $A_{\mathrm{EN}}(\pi)=0$, and we state the proposition without proof.

Proposition 3.4 Let $\pi \in \mathfrak{S}_{n}$ and let $\mathcal{T}=\Gamma(\pi) \in \mathfrak{T}_{n}$. Assume $A_{\mathrm{EN}}(\pi)=0$, then the following algorithm gives $\mathcal{T}^{\prime \prime}=\operatorname{irc}(\mathcal{T})$. Given $\lambda=\operatorname{sh}(\mathcal{T})$ and $k=\operatorname{wex}(\pi)$, let $\mathcal{T}^{\prime}$ have shape $\lambda_{1}^{k}$ and let $z\left(c_{i}\left(\mathcal{T}^{\prime}\right)\right)=z\left(c_{i}(\mathcal{T})\right)$ for all columns (and similarly for the rows). Then, let $\mathcal{T}^{\prime \prime}=\operatorname{irc}\left(\mathcal{T}^{\prime}\right)$ and increase column labels $j$ by $t\left(c_{j}(\mathcal{T})\right)$.

Example 3.5 The permutation $\pi=38652417$ has $A_{\mathrm{EN}}(\pi)=4$ and $A_{\mathrm{NE}}(\pi)=0$. Its tableau is below to the right. Removing the EN zeroes gives the second tableau, where the column exit label 7 has been reduced by 3 and 4 has been reduced by 1 . Applying irc gives the third tableau, and inserting 3 twos in the forth column and 1 in the third gives the final tableau of $\operatorname{irc}(\pi)=71653842$ to the right. To compute irc of the last tableau, just follow the tableaux below from right to left.
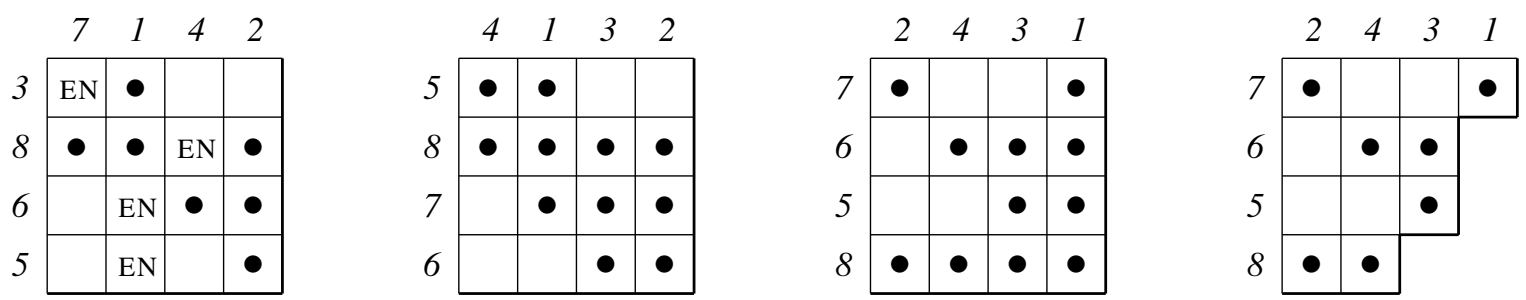

As it turns out, the processes of turning EN zeroes into NE twos and vice versa are independent processes. Combining the last two Propositions thus gives us an algorithm for computing $\operatorname{irc}(\mathcal{T})$ for any 1-hinge permutation tableau $\mathcal{T}$.

Theorem 3.6 Let $\pi \in \mathfrak{S}_{n}$ and let $\mathcal{T}=\Gamma(\pi) \in \mathfrak{T}_{n}$, with $\lambda=\operatorname{sh}(\mathcal{T}), k=\operatorname{wex}(\pi)$ and column exit labels $i_{1}<i_{2}<\ldots<i_{k}$. To compute $\mathcal{T}^{\prime \prime}=\operatorname{irc}(\mathcal{T})$, let $\mathcal{T}^{\prime}$ have shape $\lambda_{1}^{k}$ and let $z\left(c_{i}\left(\mathcal{T}^{\prime}\right)\right)=\operatorname{inv}\left(c_{i}(\mathcal{T})\right)$ for all columns (and likewise for the rows). Then, let $\mathcal{T}^{\prime \prime}=\operatorname{irc}\left(\mathcal{T}^{\prime}\right)$, increase column labels $j$ by $t\left(c_{j}(\mathcal{T})\right.$ ) and $t\left(c_{j}\left(\mathcal{T}^{\prime \prime}\right)\right)=i_{j}-j$ for all $j$. 
Proof: Removing the twos of $\mathcal{T}$ corresponds to multiplying $\pi$ by cycles on the right as in the proof of Proposition 3.3. Removing the EN zeroes corresponds to multiplying $\pi$ by cycles on the left. These processed are commutative. We can then compute irc and intepret the cycles moving from left to right as NE twos and the other cycles as EN zeroes.

Example 3.7 The permutation $\pi=38265417$ has $A_{\mathrm{EN}}(\pi)=4$ and $A_{\mathrm{NE}}(\pi)=2$. Its tableau is below to the right. Removing the EN zeroes and the twos gives the second tableau, which is the same as the second tableau in the previous example. Applying irc gives the third tableau, and inserting twos as before, as well as two EN zeroes by increasing column exit label 4 to 6 gives the final tableau of $\operatorname{irc}(\pi)=71543862$ to the right.
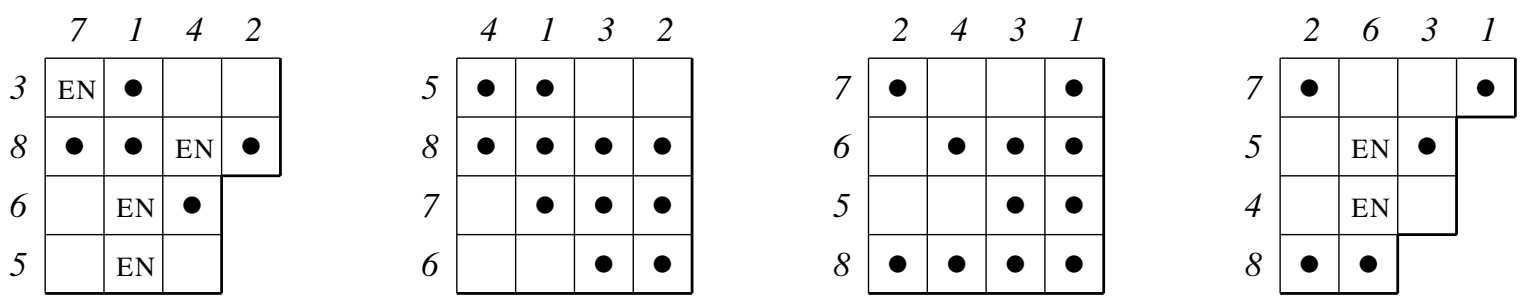

\section{The $A \leftrightarrow C$ bijection}

Steingrímsson and Williams showed that $A_{\mathrm{NN}}+A_{\mathrm{EE}}$ is equidistributed with $C_{\mathrm{NN}}+C_{\mathrm{EE}}$. We would like to show this with a simple bijection, which exchanges these statistics while preserving $A_{\mathrm{NE}}, A_{\mathrm{EN}}$ and wex. We name this bijection $\psi: \Gamma\left(\mathfrak{S}_{n}\right) \rightarrow \Gamma\left(\mathfrak{S}_{n}\right)$. To find $\psi$, we need to keep track of the relative order of the exit labels of rows and columns.

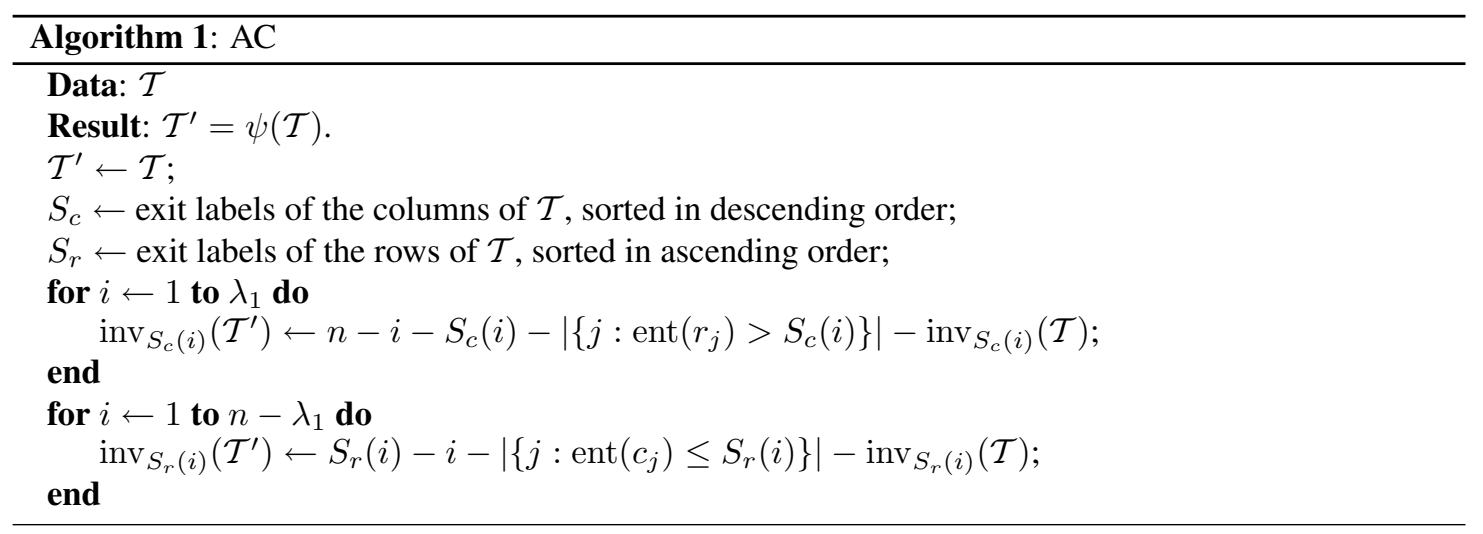

Theorem 4.1 Algorithm 1 is an involution on permutation tableaux $\mathcal{T}$ such that $\mathcal{T}^{\prime}=\psi(\mathcal{T})$ fulfills

- $A_{\mathrm{NN}}\left(\Phi\left(\mathcal{T}^{\prime}\right)\right)+A_{\mathrm{EE}}\left(\Phi\left(\mathcal{T}^{\prime}\right)\right)=C_{\mathrm{NN}}(\Phi(\mathcal{T}))+C_{\mathrm{EE}}(\Phi(\mathcal{T}))$; 
- $C_{\mathrm{NN}}\left(\Phi\left(\mathcal{T}^{\prime}\right)\right)+C_{\mathrm{EE}}\left(\Phi\left(\mathcal{T}^{\prime}\right)\right)=A_{\mathrm{NN}}(\Phi(\mathcal{T}))+A_{\mathrm{EE}}(\Phi(\mathcal{T}))$

- $A_{\mathrm{EN}}\left(\Phi\left(\mathcal{T}^{\prime}\right)\right)=A_{\mathrm{EN}}(\Phi(\mathcal{T}))$;

- $A_{\mathrm{NE}}\left(\Phi\left(\mathcal{T}^{\prime}\right)\right)=A_{\mathrm{NE}}(\Phi(\mathcal{T}))$;

- $\operatorname{sh}\left(\mathcal{T}^{\prime}\right)=\operatorname{sh}(\mathcal{T})$

Proof: It is clear that the proposed map is an involution, but there are three additional items for us to prove: that each tableau $\mathcal{T}$ is a valid input, that the obtained tableau $\mathcal{T}^{\prime}$ is valid and finally that the statistics are transformed as stated. We take these matters in order.

In the rest of the proof, we will consider rows only, but the columns are treated analogously. Now, the maximal number of rows between the entry and the exit of the path with exit label $S_{r}(i)$ is $S_{r}(i)-i-\mid\{j$ : ent $\left.\left(c_{j}\right) \leq S_{r}(i)\right\} \mid$. Since inv $S_{r}(i)$ is bounded from above by this number and from below by zero for both $\mathcal{T}$ and $\mathcal{T}^{\prime}$, it is clear that each tableau $\mathcal{T}$ is a valid input, and that $\psi(\mathcal{T})$ is a valid tableau.

Since we do not change the exit labels of the rows, except for their order, the value of $A_{\mathrm{EN}}$ stays constant. By the argument that $\operatorname{inv}_{S_{r}(i)}\left(\mathcal{T}^{\prime}\right)$ stays within the given bounds, it follows that the shape and $A_{\mathrm{NE}}$ does not change either. If $A_{\mathrm{EN}}=A_{\mathrm{NE}}=0$, the total number of inversions for $\mathcal{T}$ and $\mathcal{T}^{\prime}$ clearly is $(k-1)(n-k)$, which shows that for this case, $A_{\mathrm{EE}}+A_{\mathrm{NN}}$ and $C_{\mathrm{EE}}+C_{\mathrm{NN}}$ must exchange. But since each increase in $A_{\mathrm{NE}}$ or $A_{\mathrm{EN}}$ decreases the number of inversions equally, the exchange must still hold.

It is not too hard to deduce the following special cases of the $\psi$ involution.

Corollary 4.2 Let the extended diagonal be the usual main diagonal followed to the right by the remainder of the lowest row extending past the main diagonal. For $\mathcal{T}$ such that $A_{\mathrm{EN}}(\mathcal{T})=A_{\mathrm{NE}}(\mathcal{T})=0$, we get

$z(\operatorname{col}(i, \psi(\mathcal{T})))=\operatorname{inv}(\operatorname{col}(i, \psi(\mathcal{T})))=\min (i, \operatorname{wex}(\Phi(\mathcal{T})))-1-\operatorname{inv}(\operatorname{col}(i, \mathcal{T}))=\min (i, \operatorname{wex}(\Phi(\mathcal{T})))-1-z(\operatorname{col}(i, \mathcal{T}))$

and

$$
z(\operatorname{row}(i, \psi(\mathcal{T})))=\operatorname{inv}(\operatorname{row}(i, \psi(\mathcal{T})))=\min (i, \operatorname{wex}(\Phi(\mathcal{T})))-1-z(\operatorname{row}(i, \mathcal{T})) .
$$

Example 4.3 Consider the permutation $\pi=76813524$ with tableau as below. The number of inital zeroes is zero for exit labels $1,2,4$, one for 3 and two for 5 . Hence, in $\psi(\mathcal{T})$ we get $z(\operatorname{col}(5))=2-2=0$, $z(\operatorname{col}(4))=2-0=2, z(\operatorname{col}(3))=2-1=1, z(\operatorname{col}(2))=1-0=1$ and $z(\operatorname{col}(5))=2-2=0$.
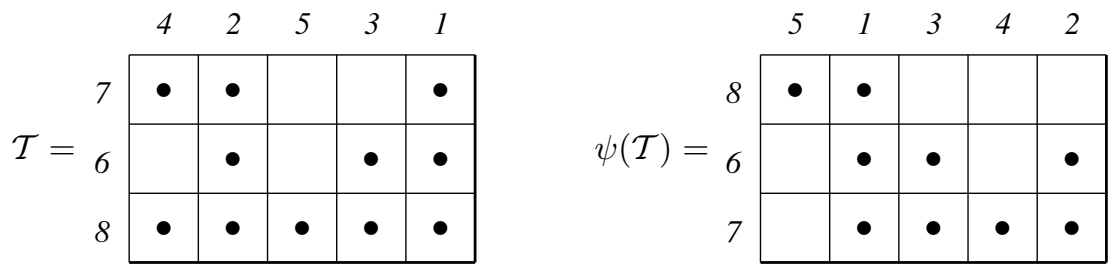
Corollary 4.4 Consider any tableau $\mathcal{T}$ such that $A_{\mathrm{NN}}(\mathcal{T})=A_{\mathrm{EE}}(\mathcal{T})=A_{\mathrm{NE}}(\mathcal{T})=0$, and split it in a lower part consisting of the bottom row, a left part where coordinates $(i, j)$ satisfy $i-j \geq n-2 \lambda_{1}$ and $i<n-\lambda_{1}$ and an upper part where $\lambda_{1}+i-j<n-\lambda_{1}$. Then, $\operatorname{inv}\left(c_{i}(\psi(\mathcal{T}))\right)$ equals the number of ones in column $i$ in the left part and $\operatorname{inv}\left(r_{i}(\psi(\mathcal{T}))\right)$ the number of ones in row $i$ in the upper part of $\mathcal{T}$.

Example 4.5 The permutation $\pi=157923468$ fulfills the conditions of the previous corollary. We take its tableau and split it.
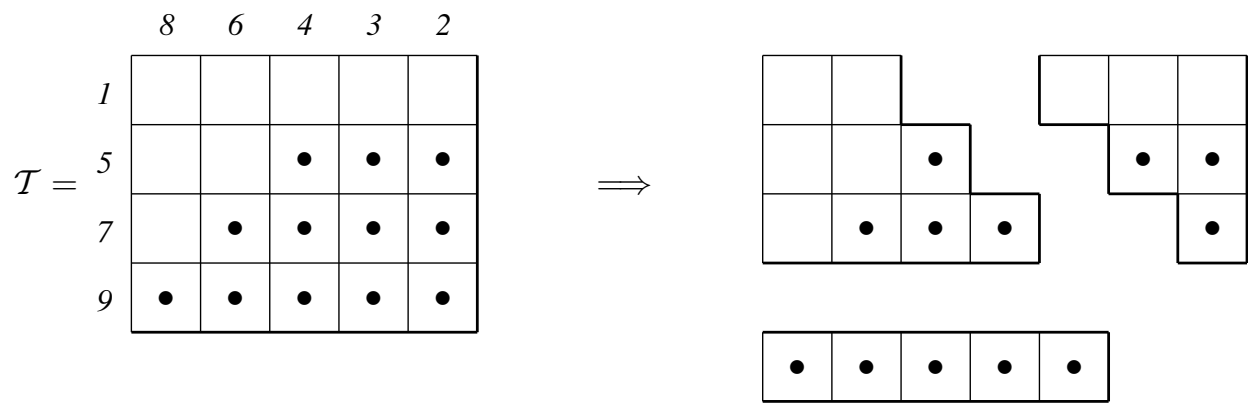

The number of ones in the left part is, from the left, 0,1,2 and 1, and the number of ones in the upper part is, from above, 0, 2 and 1 . Thus, the permutation becomes 197543628, with two inversions on 5 and one on 7, and similarly for the columns.

Corollary 4.6 Consider any tableau $\mathcal{T}$ such that $A_{\mathrm{NN}}(\mathcal{T})=A_{\mathrm{EE}}(\mathcal{T})=A_{\mathrm{EN}}(\mathcal{T})=0$. Then, the tableau of $\psi(\mathcal{T})$ contains only zeroes and the extended diagonal filled with ones, possibly pushed up by the south-east border.

Example 4.7 For the permutation 671283945 , the tableaux $\mathcal{T}=\Phi(\pi)$ and $\psi(\mathcal{T})$ become
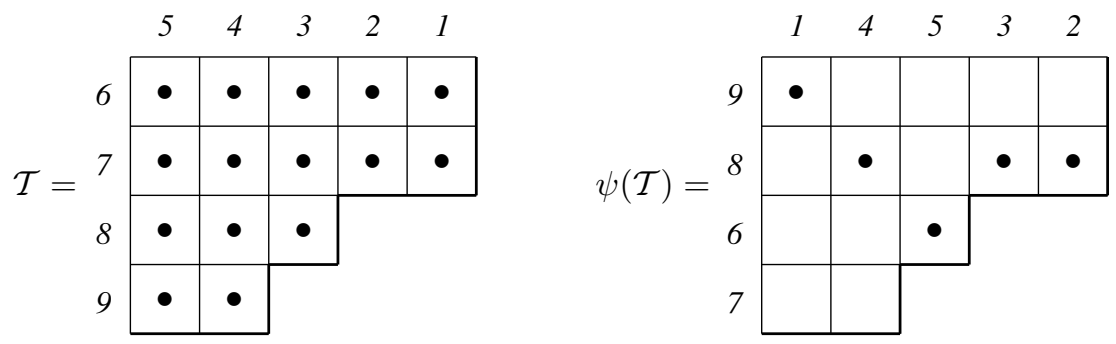

\section{Tableaux of restricted permutations}

In this section, we will enumerate some restricted sets of permutations whose 1-hinge tableaux have the maximum number of essential $1 \mathrm{~s}$ as a first step in determining the distribution of restricted permutations 
according to the number of the essential 1s of their associated tableaux. Let $M_{n}$ denote the set of permutations in $\mathfrak{S}_{n}$ whose 1-hinge tableaux have $n-1$ essential 1s (i.e. the maximum number). As we noted in the introduction, not every set of pattern restrictions refines well by the essential 1s statistic, so we find analyze some of the "nicer" cases here.

Definition 5.1 We say that a permutation $\sigma \in \mathfrak{S}_{n}$ contains a pattern $\tau$ if $\tau$ is a permutation orderisomorphic to a subsequence of $\sigma$. If $\sigma$ does not contain the pattern $\tau$, then we say that $\sigma$ avoids $\tau$. We denote the set of $\tau$-avoiding permutations in $\mathfrak{S}_{n}$ by $\mathfrak{S}_{n}(\tau)$. Given a set of patterns $T$, we let $\mathfrak{S}_{n}(T)$ be the set of permutations avoiding all patterns in $T$.

Similarly, given a pattern (or a set of patterns) $\tau$, let $M_{n}(\tau)$ denote the set of permutations in $\mathfrak{S}_{n}(\tau)$ whose 1-hinge tableaux have $n-1$ essential 1 s, i.e. a single doubly essential 1 (i.e. both the leftmost 1 in its row and the topmost 1 in its column). We then proceed to determine the structure of these tableaux to prove some enumerative results.

We note that, for simplicity, the patterns here are denoted the "old" way, without using the newer generalized-pattern hyphenated notation.

Remark 5.2 Note that any permutation $\pi \in M_{n}$ must have a 1 in upper left corner of its corresponding 1-hinge tableau. In particular, this implies that $\pi(1)>\pi(n)$. Also, the 1-hinge tableau of $\pi$ must contain at least one 1 in every row, and hence $\pi$ does not have fixed points.

Theorem 5.3 The number of permutations in $M_{n}(132,231)$ (resp. in $M_{n}(213,312)$ ) whose 1-hinge tableaux have $k$ rows (resp. $k$ columns) is equal to

$$
2^{k-1}\left(\begin{array}{c}
n-k-1 \\
k-1
\end{array}\right)-2^{k-2}\left(\begin{array}{c}
n-k-2 \\
k-2
\end{array}\right) .
$$

Proof: Any permutation in $\pi \in \mathfrak{S}_{n}(132,231)$ can be written as $\pi=\pi^{\prime} 1 \pi^{\prime \prime}$, where $\pi^{\prime}$ is a decreasing sequence and $\pi^{\prime \prime}$ is an increasing sequence. Thus, either $n=\pi(1)$ or $n=\pi(n)$, so by Remark 5.2, we have $n=\pi(1)$ for $\pi \in M_{n}(132,231)$. Therefore, the leftmost column of the 1-hinge tableau $\mathcal{T}$ of $\pi$ contains a 1 only in the first row. We also conclude that $n-1=\pi(2)$ or $n-1=\pi(n)$. In addition, since $\pi$ is a derangement and $n=\pi(1)$, it follows that $\pi(n-1)<n-1$, so $n-1$ is a column label in $\pi$.

From this, we can conclude that the first $k<\pi^{-1}(1)$ positions are excedances, and the remaining ones are deficiences, so the shape of $\mathcal{T}$ is a $k \times(n-k)$ rectangle. Further, $\operatorname{ext}\left(r_{i}\right)>\operatorname{ext}\left(r_{i+1}\right), \operatorname{ext}\left(r_{k}\right)=$ $\pi(k)>\pi(k+1)=\operatorname{ext}\left(c_{n-k}\right)$ and $\operatorname{ext}\left(c_{i}\right)>\operatorname{ext}\left(c_{i+1}\right)$ for $i \geq m$, where $m$ is the column with exit label 1. We can thus conclude that $z\left(r_{i}\right)=n-\operatorname{ext}\left(r_{i}\right)$, as well as $z\left(c_{i}\right)=\operatorname{ext}\left(c_{i}\right)-1$ for $i>m$ and zero otherwise. The $1 \mathrm{~s}$ in the tableau are thus on a northwest-southeast directed band so that no 1 has only $0 \mathrm{~s}$ in both its row and its column.

To determine such a tableau, we need to determine for each row the leftmost and rightmost position. The leftmost position in $r_{i}$ is at least one more than in row $r_{i-1}$, since $z\left(r_{i}\right)=n-\operatorname{ext}\left(r_{i}\right)>n-\operatorname{ext}\left(r_{i-1}\right)=$ $z\left(r_{i-1}\right)$, and the rightmost position changes by at most one, since two adjacent columns cannot share the same number of zeroes to the right of $m$. Further, the leftmost one of the first row and the rightmost one of the last row are fixed.

Thus, we need to pick a subset of $k-1$ columns among all columns but the first one to get the leftmost positions in rows 2 to $k$, and for each of the first $k-1$ rows, we need to determine if the rightmost 1 should be to the left of the row below or not. The number of such choices is $2^{k-1}\left(\begin{array}{c}n-k-1 \\ k-1\end{array}\right)$. However, we 
cannot leave a 1 in the southeast corner with no other $1 \mathrm{~s}$ above it or to its left, so all such tableaux must be removed. They are counted by $2^{k-2}\left(\begin{array}{c}n-k-2 \\ k-2\end{array}\right)$, and we are done.

Theorem 5.4 If $M_{n}^{k}(123,213)$ is the set of permutations in $M_{n}(123,213)$ with $k$ nonessential $1 s$, then

$$
\begin{aligned}
\left|M_{2 n}^{k}(123,213)\right| & =a(2 n-2, k)+a(2 n-3, k), \\
\left|M_{2 n+1}^{k}(123,213)\right| & =2 a(2 n-2, k)+a(2 n-3, k),
\end{aligned}
$$

where $a(n, k)=\mathrm{A} 037027(n, k)$ (see [11]), the kth entry in row $n$ of the Fibonacci-Pascal triangle.

Proof: Let $\pi \in M_{n}^{k}(123,213)$ and let $\mathcal{T}=\Phi^{-1}(\pi)$ be its permutation tableau. Simply avoidance of the both pattern implies the following: $n=\pi(1)$ or $n=\pi(2)$, and $\pi(n)=1$ or $\pi(n)=2$. Also, if $\pi(1)=n$ and $\pi(n)=1$, then the top row (labeled 1) and leftmost column (labeled $n$ ) of $\mathcal{T}$ both have a single 1 in the top left cell (labeled $(1, n)$ ). Thus, the cell labeled $(2, n-1)$ in the second row from the top and next-to-leftmost column is also a doubly essential 1, and hence, $\pi$ does not have the maximum number of essential 1s. Therefore, either $\pi(2)=n$ and $\pi(n)=1$, or $\pi(1)=n$ and $\pi(n)=2$, or $\pi(2)=n$ and $\pi(n)=2$.

Case 1. Let $n \geq 3, \pi(n)=1$ and $\pi(2)=n$. Since $\pi(n)=1$, the top row has a single 1 in the leftmost column. Since $\pi(2)=n$, the leftmost column has a 1 in row 2 and no 1 s below it. Thus, we have

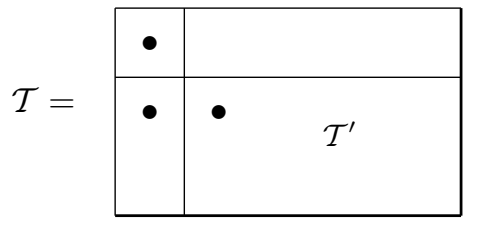

Let $\mathcal{T}^{\prime}$ be the tableau obtained by removing the top row and leftmost column of $\mathcal{T}$. Then, for $\mathcal{T}$ to have the maximum number of $1 \mathrm{~s}, \mathcal{T}^{\prime}$ must also have the maximum number of essential $1 \mathrm{~s}$, and in particular, an essential 1 in the top left corner. Moreover, the nonessential $1 \mathrm{~s}$ of $\mathcal{T}^{\prime}$ are exactly the nonessential $1 \mathrm{~s}$ of $\mathcal{T}$. Let $\pi^{\prime}=\Phi\left(\mathcal{T}^{\prime}\right)$, then $\pi(1)=\pi^{\prime}(1)+1, \pi(2)=n, \pi(i)=\pi^{\prime}(i-1)+1$ for $3 \leq i \leq n-1$, and $\pi(n)=1$. In other words, $\pi^{\prime}$ is obtained from $\pi$ by removing 1 and $n$ and subtracting 1 from each of the remaining values. Hence, $\pi^{\prime} \in M_{n-2}^{k}(123,213)$.

Case 2. Let $n \geq 3, \pi(2)=n$ and $\pi(n)=2$. Since the leftmost column has $1 \mathrm{~s}$ in the rows 1 and 2 , any 1 in row 1 induces a (nonessential) 1 in the cell directly below it. Since $\pi(n) \neq 1$, there is at least one 1 in the top row in addition to the 1 in the leftmost cell. Suppose the second leftmost 1 in the top row is in column labeled $i$. Then the path starting south at column $n$ turns east at cell $(1, n)$, south at $(1, i)$, and east at $(2, i)$. Since $\pi(n)=2$, that path exits the tableau at row 2 , so there are no $1 \mathrm{~s}$ in row 2 to the right of column $i$. Hence, there are also no $1 \mathrm{~s}$ in row 1 to the right of column $i$, so $\pi(i)=1$ and row 1 has, in fact, only two $1 \mathrm{~s}$, in the leftmost column and in column $i$. Let $\mathcal{T}^{\prime}$ be the tableau obtained by removing the top row and leftmost column of $\mathcal{T}$. Then, just as in the previous case, it is easy to see that $\mathcal{T}$ has exactly one more nonessential 1 than $\mathcal{T}^{\prime}$, namely, the 1 in the cell labeled $(2, i)$. Moreover, $\mathcal{T}^{\prime}$ also has the maximum number of essential $1 \mathrm{~s}$, and $\pi^{\prime}=\Phi\left(\mathcal{T}^{\prime}\right)$ is obtained by deleting 2 and $n$ from $\pi$ and subtracting 1 from each remaining value except 1 . Therefore, $\pi^{\prime}$ also avoids patterns 123 and 213 , and hence, $\pi^{\prime} \in M_{n-2}^{k-1}(123,213)$. 
Case 3. Let $n \geq 3, \pi(1)=n$ and $\pi(n)=2$. Since $\pi(1)=n$, the leftmost column of $\mathcal{T}$ contains a single 1 in the top row. Therefore, the leftmost column cannot be longer than the column to its right, and hence the second leftmost column has label $n-1$. Since $\mathcal{T}$ has the maximum number of essential $1 \mathrm{~s}$ and 0 in cell $(2, n)$, either $n=3$ and $\pi=312$ or there must be a 1 in the cell $(2, n-1)$. Thus, the path starting south at column $n$, turns east at cell $(1, n)$, then again south at $(1, n-1)$, and so passes through cell $(2, n-1)$. Since $\pi(n)=2$, this means that $\mathcal{T}$ has a 1 in the cell $(2, n-1)$ and only 0 s to its right. Hence, this is the single 1 in row 2 , so either $n=4$ and $\pi=4312$ or $n \geq 5$ and $\mathcal{T}$ must have a 1 in the cell $(3, n-1)$. Also, the fact that row 2 has no $1 \mathrm{~s}$ to the right of row $n-1$ means that row 1 has no $1 \mathrm{~s}$ to the right of column $n-1$, so $\pi(n-1)=1$. Finally, since $\pi(1)=n$ and $\pi$ avoids patterns 123 and 213, we must have $n-1=\pi(2)$ or $n-1=\pi(3)$. Since column $n$ has no 1 s other than in top row, $\pi(i)=n-1$ implies that $i$ is the lowest row with a 1 in column $n-1$. Since there is a 1 in cell $(3, n-1)$, we must have $n-1=\pi(3)$, so there are no $1 \mathrm{~s}$ in column $n-1$ below row 3 . Therefore, either $n=5$ and $\pi=53412$ or $n \geq 6$ and there is a 1 in cell $(3, n-2)$.

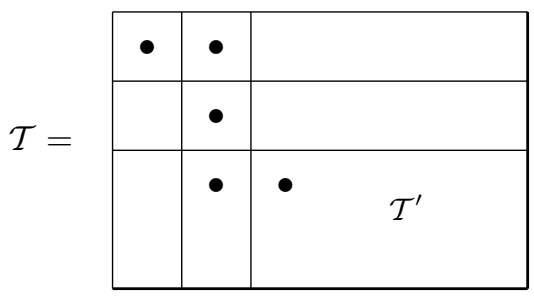

Let $\mathcal{T}^{\prime}$ be the tableau obtained by deleting the top two rows and leftmost two columns of $\mathcal{T}$, and let $\pi^{\prime}=\Phi\left(\mathcal{T}^{\prime}\right)$. Then $\mathcal{T}^{\prime}$ has the maximum number of essential $1 \mathrm{~s}$ and the same number of nonessential $1 \mathrm{~s}$ as $\mathcal{T}$, and $\pi(1)=n, \pi(2)=\pi^{\prime}(1)+2, \pi(3)=n-1, \pi(i)=\pi(i-2)+2$ for $4 \leq i \leq n-2$, and $\pi(n-1)=1, \pi(n)=2$. In other words, $\pi^{\prime}$ is obtained from $\pi$ by deleting $n, n-1,1,2$ at positions $1,3, n-1, n$, respectively, and subtracting 2 from each of the remaining values. Therefore, $\pi^{\prime}$ also avoids 123 and 213 , so $\pi^{\prime} \in M_{n-4}^{k}(123,213)$.

Thus, it is easy to see that $f(n, k)=\left|M_{n}^{k}(123,213)\right|$ satisfies the recurrence relation

$$
f(n, k)=f(n-2, k)+f(n-2, k-1)+f(n-4, k)
$$

with initial values $f(0,0)=1, f(1,0)=1, f(2,0)=1, f(3,0)=2, f(3,1)=0 f(4,0)=2$, $f(4,1)=1$, and $f(n, k)=0$ if $k<0$ or $k>(n-2) / 2$ for $n \geq 2$. A routine application of generating functions yields the theorem.

One can similarly prove the following statements. We omit the proofs in this extended abstract.

Theorem 5.5 Let $M_{n}(123,213 ; k)$ be the set of permutations in $M_{n}(123,213)$ that start with the letter $k$. Then, for $1 \leq k \leq n \leq 2 k+3$, we have

$$
\begin{aligned}
\left|M_{n}(123,213)\right| & =\mathrm{A} 002965(n+1)(\operatorname{see}[11]), \quad n \geq 0, \\
\left|M_{n}(123,213 ; k)\right| & =2^{n-k} \mathrm{~A} 002965(2 k-n-3), \quad n \geq 4,\lfloor n / 2\rfloor+2 \leq k \leq n, \\
\left|M_{n}(123,213 ; k)\right| & =1, \quad(n, k) \in\{(1,1),(2,2),(3,3),(3,2)\} .
\end{aligned}
$$

Theorem 5.6

$$
\left|M_{n}(132,213)\right|= \begin{cases}2 \cdot 3^{(n-2) / 2}-1 & \text { if } n \text { is even } \\ 3^{(n-1) / 2}-1 & \text { if } n \text { is odd }\end{cases}
$$




\section{References}

[1] A. Burstein. On some properties of permutation tableaux, Ann. Combin. 11 (2007), 355-368.

[2] R. Chapman, L. K. Williams. A conjecture of Stanley on alternating permutations, Electron. J. Combin. 14(1) (2007), \#N16.

[3] S. Corteel. Crossings and alignments of permutations, Adv. Appl. Math., to appear, arXiv:math.CO/0601469.

[4] S. Corteel, A simple bijection between permutation tableaux and permutations, preprint, arXiv:math.CO/0601469.

[5] S. Corteel, L. K. Williams. A Markov chain on permutations which projects to the PASEP (partially asymmetric exclusion process), Internat. Math. Res. Notices, 2007, article ID mm055. Also at arXiv:math.CO/0609188.

[6] S. Corteel, L. K. Williams. Tableaux combinatorics for the asymmetric exclusion process, Adv. Appl. Math. 39 (2007), no. 3, 293-310. Also at arXiv:math.CO/0602109.

[7] K. Eriksson. Strongly convergent games and Coxeter groups, Ph. D. thesis, KTH, Stockholm, 1993.

[8] A. Postnikov. Webs in totally positive Grassmann cells, manuscript, 2001.

[9] E. Steingrímsson, L. K. Williams. Permutation tableaux and permutation patterns, J. Combin. Th. Ser. A 114 (2007), 211-234.

[10] L. K. Williams. Enumeration of totally positive Grassmann cells, Adv. Math. 190 (2005), no. 2, 319-342.

[11] N. J. A. Sloane, The On-Line Encyclopedia of Integer Sequences (2008), published electronically at http://www.research.att.com/〜njas/sequences/. 
